\title{
Relations between Schoenberg Coefficients on Real and Complex Spheres of Different Dimensions
}

\author{
Pier Giovanni BISSIRI ${ }^{\dagger}$, Valdir A. MENEGATTO ${ }^{\ddagger}$ and Emilio PORCU ${ }^{\dagger \S}$ \\ † School of Mathematics \& Statistics, Newcastle University, \\ Newcastle Upon Tyne, NE1 7RU, UK \\ E-mail: pier-giovanni.bissiri@newcastle.ac.uk,emilio.porcu@newcastle.ac.uk \\ ‡ Instituto de Ciências Matemáticas e de Computação, Universidade de São Paulo, \\ Caixa Postal 668, 13560-970, São Carlos - SP, Brazil \\ E-mail: menegatt@icmc.usp.br \\ $\S$ Department of Mathematics, University of Atacama, Copiapó, Chile
}

Received July 25, 2018, in final form January 18, 2019; Published online January 23, 2019

https://doi.org/10.3842/SIGMA.2019.004

\begin{abstract}
Positive definite functions on spheres have received an increasing interest in many branches of mathematics and statistics. In particular, the Schoenberg sequences in the spectral representation of positive definite functions have been studied by several mathematicians in the last years. This paper provides a set of relations between Schoenberg sequences defined over real as well as complex spheres of different dimensions. We illustrate our findings describing an application to strict positive definiteness.
\end{abstract}

Key words: positive definite; Schoenberg pair; spheres; strictly positive definite

2010 Mathematics Subject Classification: 33C45; 42A16; 42A82; 42C10

\section{Introduction}

Positive definite functions on real and complex spheres have a long history, that starts with the seminal paper by Schoenberg [41]. Positive definiteness is crucial to many branches of mathematical analysis $[4,5,9,17,21,22,23,24,33,34,36,41]$ and statistics $[3,7,10,11,12$, $13,14,18,25,26,27,28,29,30,31,38,40]$. Recent reviews on positive definite functions on either spheres or product spaces involving spheres can be found in [19] and in [39] as well.

Fourier analysis on spheres is related to the so called Schoenberg sequences (also called sequences of Schoenberg coefficients in [15]) that are related to the dimension where any positive definite function on real or complex spheres is defined. There has been a recent interest on Schoenberg sequences, especially after the list of research problems in [19] and in [39]. Recursive relations between Schoenberg coefficients on $d$-dimensional spheres have been first proposed by [19]. Fiedler [16] has then solved an open problem in [19], related to other types of recursions involving Schoenberg coefficients. Ziegel [44] has used Schoenberg sequences to find the convolution roots of positive definite functions on spheres and illustrated the differentiability properties of positive definite functions on spheres through their Schoenberg sequences in [42]. Recently, Arafat et al. [2] have solved Gneiting's research problem number 3 making extensive use of Schoenberg sequences. Projections from Hilbert into finite-dimensional spheres have been considered in [38]. Finally, Schoenberg sequences have been shown to be central to the study of geometric properties of Gaussian fields on spheres [29] or spheres cross time [14].

Literature on complex spheres has been sparse. After the tour de force in [35] there has been a recent interest on complex spheres as reported from [6] and in [32]. 
This paper is about Schoenberg sequences on spheres of $\mathbb{R}^{d}$ and $\mathbb{C}^{q}$, respectively. Specifically, we show recursive relations that have been lacking from the previously mentioned literature. Section 2 deals with real-valued $d$-dimensional spheres. Section 3 is instead related to complex spheres. Some implications in terms of strict positive definiteness are provided in Section 4 . The paper ends with a discussion.

\section{Schoenberg sequences on real spheres}

\subsection{Background and notation}

For a positive integer $d$, let $\mathbb{S}^{d}=\left\{\mathbf{x} \in \mathbb{R}^{d+1},\|\mathbf{x}\|=1\right\}$ denote the $d$-dimensional unit sphere embedded in $\mathbb{R}^{d+1}$, with $\|\cdot\|$ being the Euclidean norm. We define the great-circle distance $\theta: \mathbb{S}^{d} \times \mathbb{S}^{d} \rightarrow[0, \pi]$ as the continuous mapping defined through

$$
\theta\left(\mathbf{x}_{1}, \mathbf{x}_{2}\right)=\arccos \left(\mathbf{x}_{1}^{\top} \mathbf{x}_{2}\right) \in[0, \pi]
$$

for $\mathbf{x}_{1}, \mathbf{x}_{2} \in \mathbb{S}^{d}$, where $\top$ is the transpose operator. A mapping $C: \mathbb{S}^{d} \times \mathbb{S}^{d} \rightarrow \mathbb{R}$ that satisfies

$$
\sum_{i, j=1}^{n} c_{i} c_{j} C\left(\mathbf{x}_{i}, \mathbf{x}_{j}\right) \geq 0
$$

for all $n \geq 1$, distinct points $\mathbf{x}_{1}, \mathbf{x}_{2}, \ldots, \mathbf{x}_{n}$ on $\mathbb{S}^{d}$ and real numbers $c_{1}, \ldots, c_{n}$, is called positive definite. Further, if the inequality is strict, unless the vector $\left(c_{1}, \ldots, c_{n}\right)^{\top}$ is the zero vector, then it is called strictly positive definite (see [8] and references therein). If, in addition,

$$
C\left(\mathbf{x}_{1}, \mathbf{x}_{2}\right)=\psi\left(\theta\left(\mathbf{x}_{1}, \mathbf{x}_{2}\right)\right), \quad \mathbf{x}_{i} \in \mathbb{S}^{d}, \quad i=1,2,
$$

for some mapping $\psi:[0, \pi] \rightarrow \mathbb{R}$, then $C$ is called a geodesically isotropic covariance by [39]. With no loss of generality, we assume through the paper that the function $\psi$ is continuous along with the normalization $\psi(0)=1$.

Gneiting [19] calls $\Psi_{d}$ the class of continuous functions $\psi:[0, \pi] \rightarrow \mathbb{R}$ with $\psi(0)=1$ such that the function $C$ in equation (2.1) is positive definite. The inclusions $\Psi_{d} \supset \Psi_{d+1}, d \geq 1$, are known to be strict. Following [41], for every continuous function $\psi:[0, \pi] \rightarrow \mathbb{R}$ with $\psi(0)=1$, and every integer $d \geq 2$, define

$$
b_{n, d}=\kappa(n, d) \int_{0}^{\pi} \psi(\theta) C_{n}^{(d-1) / 2}(\cos \theta)(\sin \theta)^{d-1} \mathrm{~d} \theta,
$$

where, for any $\lambda>0, C_{n}^{\lambda}$ denotes the $n$-th Gegenbauer polynomial of order $\lambda[1]$, and

$$
\kappa(n, d)=\frac{(2 n+d-1)\left(\Gamma((d-1) / 2)^{2}\right.}{2^{3-d} \pi \Gamma(d-1)} .
$$

Moreover, we define

$$
b_{0,1}=\frac{1}{\pi} \int_{0}^{\pi} \psi(\theta) \mathrm{d} \theta, \quad b_{n, 1}=\frac{2}{\pi} \int_{0}^{\pi} \cos (n \theta) \psi(\theta) \mathrm{d} \theta, \quad n \geq 1 .
$$

Note that in the cases $d=1$ (the circle) and $d=2$ (the unit sphere of $\mathbb{R}^{3}$ ), Gegenbauer polynomials simplify to Chebyshev and Legendre polynomials [1], respectively.

The coefficient sequences $\left\{b_{n, d}\right\}_{n=0}^{\infty}$ play a crucial role in the spectral representations for positive definite functions on spheres, which are the equivalent of Bochner and Schoenberg's theorems in Euclidean spaces (see [15] with the references therein) and are provided by [41], 
who shows that a mapping $\psi:[0, \pi] \rightarrow \mathbb{R}$ belongs to the class $\Psi_{d}$ if and only if it can be uniquely written as

$$
\psi(\theta)=\sum_{n=0}^{\infty} b_{n, d} c_{n}^{(d-1) / 2}(\cos \theta), \quad \theta \in[0, \pi]
$$

where $c_{n}^{\lambda}$ denotes the normalized $\lambda$-Gegenbauer polynomial of degree $n$, namely,

$$
c_{n}^{\lambda}(u)=\frac{C_{n}^{\lambda}(u)}{C_{n}^{\lambda}(1)}, \quad u \in[-1,1],
$$

and $\left\{b_{n, d}\right\}_{n=0}^{\infty}$ is a probability mass sequence. The series (2.5) is known to be uniformly convergent. We follow [15] when calling the sequence $\left\{b_{n, d}\right\}_{n=0}^{\infty}$ in (2.5) the $d$-Schoenberg sequence of coefficients, to emphasize the dependence on the index $d$ in the class $\Psi_{d}$. Accordingly, we say that $\left(\psi,\left\{b_{n, d}\right\}\right)$ is a uniquely determined $d$-Schoenberg pair if $\psi$ belongs to the class $\Psi_{d}$ and admits the expansion (2.5) with $d$-Schoenberg sequence $\left\{b_{n, d}\right\}_{n=0}^{\infty}$.

The following recursive relations among the coefficients $b_{n, d}$ and $b_{n, d+2}$ attached to a $d$ Schoenberg pair $\left(\psi,\left\{b_{n, d+2}\right\}\right)$ (see [19, Corollary 1])

$$
\begin{aligned}
& b_{0,3}=b_{0,1}-\frac{1}{2} b_{2,1}, \\
& b_{n, 3}=\frac{1}{2}(n+1)\left(b_{n, 1}-b_{n+2,1}\right), \quad n \geq 1, \\
& b_{n, d+2}=\frac{(n+d-1)(n+d)}{d(2 n+d-1)} b_{n, d}-\frac{(n+1)(n+2)}{d(2 n+d+3)} b_{n+2, d}, \quad d \geq 2, \quad n \geq 0,
\end{aligned}
$$

have actually opened for challenging questions. Fiedler [16] has shown relationships between sequences $\left\{b_{n, 2 d+1}\right\}_{n=0}^{\infty}$ and $\left\{b_{n, 1}\right\}_{n=0}^{\infty}$, on the one hand, and sequences $\left\{b_{n, 2 d}\right\}_{n=0}^{\infty}$ and $\left\{b_{n, 2}\right\}_{n=0}^{\infty}$, on the other. Proposition 1 in [2] encompasses Fiedler's result and provides a relation between the sequences $\left\{b_{n, d}\right\}_{n=0}^{\infty}, d>1$, and $\left\{b_{n, 1}\right\}_{n=0}^{\infty}$. A projection operator relating Schoenberg sequences on Hilbert spheres with $d$-Schoenberg sequences has been proposed by [38]. Yet, there are some relations that have not been discovered and these will be illustrated throughout.

\subsection{Results}

We start with a very simple result, that we report formally for the convenience of the reader.

Proposition 2.1. Let $d$, $d^{\prime}$ be positive integers, with $d>d^{\prime}$. If $\left(\psi,\left\{b_{n, d}\right\}\right)$ is a d-Schoenberg pair, then the $d^{\prime}$-Schoenberg sequence of coefficients of $\psi$ is uniquely determined as follows.

(i) For $d^{\prime} \geq 2$,

$$
b_{n, d^{\prime}}=\frac{\kappa\left(n, d^{\prime}\right)}{C_{n}^{(d-1) / 2}(1)} \sum_{n=0}^{\infty} b_{n, d} \int_{0}^{\pi} C_{n}^{(d-1) / 2}(\cos \theta) C_{n}^{\left(d^{\prime}-1\right) / 2}(\cos \theta) \mathrm{d} \theta,
$$

with $\kappa(n, d)$ as defined in $(2.3)$.

(ii) For $d^{\prime}=1$,

$$
\begin{aligned}
& b_{0,1}=\frac{1}{\pi} \sum_{n=0}^{\infty} b_{n, d} \int_{0}^{\pi} c_{n}^{(d-1) / 2}(\cos \theta) \mathrm{d} \theta \\
& b_{n, 1}=\frac{2}{\pi} \sum_{n=0}^{\infty} b_{n, d} \int_{0}^{\pi} c_{n}^{(d-1) / 2}(\cos \theta) \cos (n \theta) \mathrm{d} \theta, \quad n \geq 1
\end{aligned}
$$


Proof. The identity (2.9) is obtained substituting (2.5) into (2.2), whereas the identities (2.10) are obtained substituting (2.5) into (2.4). In both cases, exchanging integral and series is allowed owing to both bounded and uniform convergence of the series (2.5).

We are not aware of any closed-form expression for the integrals appearing in (2.10) and (2.9), and therefore of the relationships between the sequences $\left\{b_{n, d}\right\}_{n=0}^{\infty}$ and $\left\{b_{n, d^{\prime}}\right\}_{n=0}^{\infty}$ attached to a $d^{\prime}$-Schoenberg pair $\left(\psi, b_{n, d^{\prime}}\right)$, apart from the specific case where $d^{\prime}=d+2$. Indeed, [19] provides a closed-form expression for $\left\{b_{n, d+2}\right\}_{n=0}^{\infty}$ as a function of $\left\{b_{n, d}\right\}_{n=0}^{\infty}$ that is given by (2.6)-(2.8). Our first main results provide an explicit expression for the inverse function

Theorem 2.2. If $\left(\psi,\left\{b_{n, 3}\right\}\right)$ is a 3-Schoenberg pair, then the 1-Schoenberg sequence of coefficients of $\psi$ is given by

$$
\begin{aligned}
b_{0,1} & =\sum_{j=0}^{\infty} \frac{1}{2 j+1} b_{2 j, 3}, \\
b_{n, 1} & =\sum_{j=0}^{\infty} \frac{2}{n+2 j+1} b_{n+2 j, 3}, \quad n \geq 1 .
\end{aligned}
$$

Proof. From identity $(2.7)$, if $\left(\psi,\left\{b_{n, 3}\right\}\right)$ is a 3 -Schoenberg pair, we have that

$$
\frac{2}{n+1} b_{n, 3}=b_{n, 1}-b_{n+2,1}, \quad n \geq 1 .
$$

Hence, for every nonnegative integer $j$, and for any positive integer $n$,

$$
\frac{2}{n+2 j+1} b_{n+2 j, 3}=b_{n+2 j, 1}-b_{n+2 j+2,1} \text {. }
$$

Summing up both sides of (2.13) from 0 to $m$, we obtain

$$
\sum_{j=0}^{m} \frac{2}{n+2 j+1} b_{n+2 j, 3}=\sum_{j=0}^{m}\left(b_{n+2 j, 1}-b_{n+2 j+2,1}\right), \quad m \geq 1 .
$$

We now use the fact that the right-hand side in equation (2.13) is telescopic. Hence, (2.14) can be written as

$$
\sum_{j=0}^{m} \frac{2}{n+2 j+1} b_{n+2 j, 3}=b_{n, 1}-b_{n+2 m+2,1}, \quad m \geq 1 .
$$

Since $\psi$ belongs to $\Psi_{1}$, the series $\sum_{n=0}^{\infty} b_{n, 1}$ converges to 1 and, therefore, the sequence $\left\{b_{n, 1}\right\}_{n=0}^{\infty}$ converges to zero. We can thus take the limit for $m \rightarrow \infty$ in equation (2.15) and this will provide (2.12). In particular, we now take $n=2$ to deduce that $b_{2,1}=2 \sum_{j=1}^{\infty} b_{2 j, 3} /\{1+2 j\}$ which combined with (2.6) yields (2.11).

We are now able to provide an extension of Theorem 2.2 for $d>3$. For a positive integer $m$ and $x>0,(x)^{m}$ will denote the standard rising factorial (Pochhammer symbol).

Theorem 2.3. Let $d \geq 2$ be a positive integer. If $\left(\psi,\left\{b_{n, d+2}\right\}\right)$ is a $(d+2)$-Schoenberg pair, then the $d$-Schoenberg sequence of coefficients $\left\{b_{n, d}\right\}_{n=0}^{\infty}$ of $\psi$ is given by

$$
b_{n, d}=\sum_{j=0}^{\infty} w_{j, n, d} b_{n+2 j, d+2}, \quad n \geq 1,
$$


where

$$
\begin{aligned}
& w_{0, n, d}=\frac{d(2 n+d-1)}{(n+d-1)(n+d)}, \\
& w_{j, n, d}=d(2 n+d-1) \frac{(n / 2+1 / 2)^{(j)}(n / 2+1)^{(j)}}{(n / 2+(d-1) / 2)^{(j+1)}(n / 2+d / 2)^{(j+1)}}, \quad j \geq 1 .
\end{aligned}
$$

Proof. We give a constructive proof. Define

$$
a_{n, d}:=\frac{d(2 n+d-1)}{(n+d-1)(n+d)}, \quad u_{n, d}:=2 n+d-1, \quad v_{n, d}:=\frac{(n+1)(n+2)}{(n+d-1)(n+d)} .
$$

We can now rewrite equation (2.8) as

$$
a_{n, d} b_{n, d+2}=b_{n, d}-\frac{u_{n, d}}{u_{n+2, d}} v_{n, d} b_{n+2, d}, \quad n \geq 0 .
$$

Identity (2.16) shows that for every pair of nonnegative integers $(j, n)$, it is true that

$$
a_{n+2 j, d} b_{n+2 j, d+2}=b_{n+2 j, d}-\frac{u_{n+2 j, d}}{u_{n+2 j+2, d}} v_{n+2 j, d} b_{n+2 j+2, d} .
$$

Multiplying each side of $(2.17)$ by $\left(u_{n, d} / u_{n+2 j, d}\right) \prod_{l=0}^{j-1} v_{n+2 l, d}$ and summing up both sides from 0 to $m$, we obtain

$$
\begin{aligned}
& \sum_{j=0}^{m}\left(\prod_{l=0}^{j-1} v_{n+2 l, d}\right) \frac{u_{n, d}}{u_{n+2 j, d}} a_{n+2 j, d} b_{n+2 j, d+2} \\
& \quad=u_{n, d} \sum_{j=0}^{m}\left(\prod_{l=0}^{j-1} v_{n+2 l, d}\right)\left(\frac{b_{n+2 j, d}}{u_{n+2 j, d}}-\frac{b_{n+2 j+2, d}}{u_{n+2 j+2, d}} v_{n+2 j, d}\right) .
\end{aligned}
$$

Since the sum in the right-hand side is telescopic, we are left with

$$
\begin{aligned}
& \sum_{j=0}^{m}\left(\prod_{l=0}^{j-1} v_{n+2 l, d}\right) \frac{u_{n, d}}{u_{n+2 j, d}} a_{n+2 j, d} b_{n+2 j, d+2} \\
& \quad=b_{n, d}-\frac{u_{n, d}}{u_{n+2 m+2, d}}\left(\prod_{l=0}^{m} v_{n+2 l, d}\right) b_{n+2 j+2, d} .
\end{aligned}
$$

At this stage, note that

$$
v_{n, d}-1=-(d-2) \frac{2 n+d+1}{(n+d-1)(n+d)} \leq-(d-2) \frac{1}{n+d-1}, \quad n \geq 0 .
$$

We can now show that $\prod_{l=0}^{\infty} v_{n+2 l, d} \in\{0,1\}$. Indeed, if $d=2$, then $v_{n, d}=1$ for each $n \geq 0$ and, therefore, $\prod_{l=0}^{\infty} v_{n+2 l, 2}=1$. If $d>2$, then by $(2.19)$

$$
\begin{aligned}
\prod_{l=0}^{m} v_{n+2 l, d} & =\exp \left[\sum_{l=0}^{m} \log \left(v_{n+2 l, d}\right)\right] \\
& \leq \exp \left[\sum_{l=0}^{m}\left(v_{n+2 l, d}-1\right)\right] \leq \exp \left[-(d-2) \sum_{l=0}^{m} \frac{1}{n+2 l+d-1}\right]
\end{aligned}
$$


and, therefore, $\prod_{l=0}^{\infty} v_{n+2 l, d}=0$. Since $\psi \in \Psi_{d}$, the sequence $\left\{b_{n, d}\right\}_{n=0}^{\infty}$ converges to zero, while

$$
\lim _{m \rightarrow \infty} \frac{u_{n, d}}{u_{n+2 m+2, d}}=0, \quad n \geq 0 .
$$

So, letting $m \rightarrow \infty$ in $(2.2)$ yields

$$
b_{n, d}=\sum_{j=0}^{\infty}\left(\prod_{l=0}^{j-1} v_{n+2 l, d}\right) \frac{u_{n, d}}{u_{n+2 j, d}} a_{n+2 j, d} b_{n+2 j, d+2}, \quad n \geq 0 .
$$

Finally, direct computation shows that for $n \geq 0$ and $j \geq 1$,

$$
\prod_{l=0}^{j-1} v_{n+2 l, d}=\frac{(n / 2+1 / 2)^{(j)}(n / 2+1)^{(j)}}{(n / 2+(d-1) / 2)^{(j)}(n / 2+d / 2)^{(j)}},
$$

and

$$
\frac{u_{n, d}}{u_{n+2 j, d}} a_{n+2 j, d}=\frac{d(2 n+d-1)}{(n+2 j+d-1)(n+2 j+d)} .
$$

The proof is completed.

\section{Schoenberg sequences on complex spheres}

In analogy with the results obtained in Section 2, we consider similar results on complex spheres.

\subsection{Background and notation}

For a positive integer $q$, denote by $\Omega_{2 q}$ the unit sphere in $\mathbb{C}^{q}$. A mapping $C: \Omega_{2 d} \times \Omega_{2 q} \rightarrow \mathbb{C}$ is positive definite if

$$
\sum_{i, j=1}^{n} c_{i} \bar{c}_{j} C\left(\mathbf{z}_{i}, \mathbf{z}_{j}\right) \geq 0
$$

for all $n \geq 1$, distinct points $\mathbf{z}_{1}, \ldots, \mathbf{z}_{n}$ of $\Omega_{2 q}$ and complex numbers $c_{1}, \ldots, c_{n}$. Let "." denote the usual inner product in $\mathbb{C}^{q}$. If $q \geq 2$ and $B[0,1]=\{z \in \mathbb{C}: z \cdot z \leq 1\}$, the function $C$ is called isotropic if

$$
C\left(\mathbf{z}_{1}, \mathbf{z}_{2}\right)=\varphi\left(\mathbf{z}_{1} \cdot \mathbf{z}_{2}\right), \quad \mathbf{z}_{1}, \mathbf{z}_{2} \in \Omega_{2 q}
$$

for some function $\varphi: B[0,1] \rightarrow \mathbb{C}$. This nomenclature is not universal but it is quite adequate in our setting. Observe that in the case $q=1$, if $z, w \in \Omega_{2}$, then $z \cdot z \in \Omega_{2}$. Hence, the previous definition becomes an extreme case once the domain of $\varphi$ needs to be $\Omega_{2}$ itself.

Keeping the analogy with the previous section, for $q \geq 2$, we call $\Upsilon_{2 q}$ the class of continuous functions $\varphi$, with $\varphi(1)=1$ such that $C$ in (3.1) is positive definite. We also denote by $\Upsilon_{2 q}^{+}$ the class of functions $\varphi$ belonging to $\Upsilon_{2 q}$ such that $C$ in (3.1) is strictly positive definite. Both classes $\Upsilon_{2 q}$ and $\Upsilon_{2 q}^{+}$are nested, that is, if $q \leq q^{\prime}$, then $\Upsilon_{2 q^{\prime}} \subset \Upsilon_{2 q}$ and $\Upsilon_{2 q^{\prime}}^{+} \subset \Upsilon_{2 q}^{+}$.

To present the characterization of the class $\Upsilon_{2 q}$ described in [37], we denote by $R_{m, n}^{q-2}$ the disk polynomial of bi-degree $(m, n)$ with respect to the nonnegative integer $q-2$. The set $\left\{R_{m, n}^{q-2}: m, n=0,1, \ldots\right\}$ is a complete orthogonal system in $L^{2}\left(B[0,1], \nu_{q-2}\right)$, with

$$
\mathrm{d} \nu_{q-2}(z)=\frac{q-1}{\pi}\left(1-|z|^{2}\right)^{q-2} \mathrm{~d} x \mathrm{~d} y, \quad z=x+\mathrm{i} y \in B[0,1] .
$$


In particular,

$$
\int_{B[0,1]} R_{m, n}^{q-2}(z) \overline{R_{k, l}^{q-2}(z)} \mathrm{d} \nu_{q-2}(z)=\frac{\delta_{m k} \delta_{n l}}{h_{m, n}^{q-2}},
$$

where

$$
h_{m, n}^{q-2}=\frac{m+n+q-1}{q-1}\left(\begin{array}{c}
m+q-2 \\
q-2
\end{array}\right)\left(\begin{array}{c}
n+q-2 \\
q-2
\end{array}\right) .
$$

Expressions and main properties of disk polynomials can be found in [43] and in references quoted there. We recall the following recursion satisfied for every $z$ in $B[0,1], m \geq 1$ and $n \geq 0$ [35]:

$$
\left(1-|z|^{2}\right) R_{m-1, n}^{q-1}(z)=\frac{q-1}{m+n+q-1}\left[R_{m-1, n}^{q-2}(z)-R_{m, n+1}^{q-2}(z)\right] .
$$

For every continuous function $\varphi: B[0,1] \rightarrow \mathbb{C}$ and every triplet $(m, n, q)$ of nonnegative integers, we can define

$$
a_{m, n}^{q-2}:=h_{m, n}^{q-2} \int_{B[0,1]} \varphi(z) \overline{R_{m, n}^{q-2}(z)} \mathrm{d} \nu_{q-2}(z) .
$$

The functions belonging to the class $\Upsilon_{2 q}$ are uniquely characterized through the expansion [37]

$$
\varphi(z)=\sum_{m, n=0}^{\infty} a_{m, n}^{q-2} R_{m, n}^{q-2}(z), \quad z \in B[0,1]
$$

where $a_{m, n}^{q-2} \geq 0, m, n \in \mathbb{Z}_{+}$and $\sum_{m, n=0}^{\infty} a_{m, n}^{q-2}=1$. Following Section 2, we finally define a $2 q$ Schoenberg pair $\left(\varphi,\left\{a_{m, n}^{q-2}\right\}\right)$ any function belonging to the class $\Upsilon_{2 q}$ with expansion defined according to (3.7). In this case, the double sequence $\left\{a_{m, n}^{q-2}\right\}_{m, n=0}^{\infty}$ will be called the $2 q$-Schoenberg sequence of coefficients of $\varphi$.

\subsection{Results}

Since the classes $\Upsilon_{2 q}$ are nested, here we prove a recursive relation among the coefficients $a_{m, n}^{q-1}$ and $a_{m, n}^{q-2}$ attached to a $2(q+1)$ Schoenberg pair $\left(\varphi,\left\{a_{m, n}^{q-1}\right\}\right)$ that resembles $(2.8)$.

Proposition 3.1. If $\left(\varphi,\left\{a_{m, n}^{q-1}\right\}\right)$ is a $2(q+1)$-Schoenberg pair, then for $m-1, n \geq 0$,

$$
a_{m-1, n}^{q-1}=\frac{(m+q-2)(n+q-1)}{(q-1)(m+n+q-2)} a_{m-1, n}^{q-2}-\frac{m(n+1)}{(q-1)(m+n+q)} a_{m, n+1}^{q-2} .
$$

Proof. Equation (3.4) shows that

$$
\begin{aligned}
h_{m-1, n}^{q-2} & =\frac{(m+n+q-2) m(n+1)}{(m+n+q)(m+q-2)(n+q-1)} h_{m, n+1}^{q-2}, \quad m-1, n \geq 0, \\
h_{m-1, n}^{q-2} & =\frac{(m+n+q-2) q(q-1)^{2}}{(m+n+q-1) q(m+q-2)(n+q-1)} h_{m-1, n}^{q-1}, \quad m-1, n \geq 0 .
\end{aligned}
$$

We now multiply both sides of (3.5) by $h_{m-1, n}^{q-2} \varphi(z)$ and integrate with respect to the measure $\nu_{\alpha}$ defined in (3.3). After we use (3.6) and (3.9), we obtain

$$
\begin{aligned}
h_{m-1, n}^{q-2} & \int_{B[0,1]}\left(1-|z|^{2}\right) R_{m-1, n}^{q-1}(z) f \varphi(z) \mathrm{d} \nu_{q-2}(z) \\
\quad & =\frac{q-1}{m+n+q-1}\left[a_{m-1, n}^{q-2}-\frac{(m+n+q-2) m(n+1)}{(m+n+q)(m+q-2)(n+q-1)} a_{m, n+1}^{q-2}\right] .
\end{aligned}
$$


However, equation (3.2) yields the equality

$$
\left(1-|z|^{2}\right) d \nu_{q-2}=\frac{q-1}{q} d \nu_{q-1} .
$$

Therefore, by (3.10) and (3.6), the left-hand side of (3.11) is equal to

$$
\frac{(m+n+q-2)(q-1)^{2}}{(m+n+q-1)(m+q-2)(n+q-1)} a_{m-1, n}^{q-1},
$$

so that (3.11) becomes

$$
\frac{(m+n+q-2)(q-1)}{(m+q-2)(n+q-1)} a_{m-1, n}^{q-1}=a_{m-1, n}^{q-2}-\frac{(m+n+q-2) m(n+1)}{(m+n+q)(m+q-2)(n+q-1)} a_{m, n+1}^{q-2} .
$$

This yields (3.8).

Here is the main result of the section.

Theorem 3.2. If $\left(\varphi,\left\{a_{m, n}^{q-1}\right\}\right)$ is a $2(q+1)$-Schoenberg pair, then the 2q-Schoenberg sequence of coefficients $\left\{a_{m, n}^{q-2}\right\}_{m, n=0}^{\infty}$ of $\varphi$ is given by

$$
a_{m, n}^{q-2}=\sum_{j=0}^{\infty} v_{j, m+1, n}^{q-2} a_{m+j, n+j}^{q-1}, \quad m, n \geq 0,
$$

where

$$
v_{j, m, n}^{q-2}:=\frac{m^{(j)}(n+1)^{(j)}(m+n+q-2)}{(m+q-2)^{(j)}(n+q-1)^{(j)}(m+n+2 j+q-2)}, \quad j \geq 0 .
$$

Proof. First of all we introduce the following notations:

$$
\begin{aligned}
& u_{m, n}^{q-2}:=\frac{(q-1)(m+n+q-2)}{(m+q-2)(n+q-1)}, \quad m, n \geq 0, \\
& w_{m, n}^{q-2}:=\frac{(m+n+q-2) m(n+1)}{(m+q-2)(n+q-1)(m+n+q)}, \quad m, n \geq 0 .
\end{aligned}
$$

In this way, (3.8) becomes

$$
u_{m, n}^{q-2} a_{m-1, n}^{q-1}=a_{m-1, n}^{q-2}-w_{m, n}^{q-2} a_{m, n+1}^{q-2}, \quad m-1, n \geq 0 .
$$

By (3.13), we have that for every triplet $(j, m, n)$ of nonnegative integers,

$$
u_{m+j, n+j}^{q-2} a_{m+j-1, n+j}^{q-1}=a_{m+j-1, n+j}^{q-2}-w_{m+j, n+j}^{q-2} a_{m+j, n+j+1}^{q-2} .
$$

Now, we can multiply each side of (3.14) by the product $\prod_{l=1}^{j} w_{m+l-1, n+l-1}^{q-2}$ and sum up each side from 0 to $k$, obtaining that

$$
\begin{aligned}
& \sum_{j=0}^{k}\left(\prod_{l=1}^{j} w_{m+l-1, n+l-1}^{q-2}\right) u_{m+j, n+j}^{q-2} a_{m+j-1, n+j}^{q-1} \\
& \quad=\sum_{j=0}^{k}\left(\prod_{l=1}^{j} w_{m+l-1, n+l-1}^{q-2}\right)\left(a_{m+j-1, n+j}^{q-2}-w_{m+j, n+j}^{q-2} a_{m+j, n+j+1}^{q-2}\right)
\end{aligned}
$$


Since the sum in the right-hand side is telescopic, we are reduced to

$$
\begin{aligned}
& \sum_{j=0}^{k}\left(\prod_{l=1}^{j} w_{m+l-1, n+l-1}^{q-2}\right) u_{m+j, n+j}^{q-2} a_{m+j-1, n+j}^{q-1} \\
& \quad=a_{m-1, n}^{q-2}-\left(\prod_{j=0}^{k} w_{m+j, n+j}^{q-2}\right) a_{m+k, n+k+1}^{q-2} .
\end{aligned}
$$

Since

$$
\begin{aligned}
& \prod_{j=0}^{k} w_{m+j, n+j}^{q-2} \\
& \quad \leq \exp \left[-\sum_{j=0}^{k}\left(\frac{q-2}{m+j+q-2}+\frac{q-2}{n+j+q-1}+\frac{2}{m+n+2 j+q}\right)\right], \quad k \geq 0,
\end{aligned}
$$

we end up with

$$
\prod_{j=0}^{\infty} w_{m+j, n+j}^{q-2}=0
$$

Moreover, since

$$
\sum_{k=0}^{\infty} a_{l+k, j+k+1}^{q-2} \leq \sum_{m, n=0}^{\infty} a_{m, n}^{q-2}<\infty, \quad j, l \geq 0,
$$

we have that $\lim _{k \rightarrow \infty} a_{l+k, j+k+1}^{q-2}=0$, for $j, l \geq 0$. Therefore, letting $k \rightarrow \infty$, (3.15) leads to

$$
a_{m-1, n}^{q-2}=\sum_{j=0}^{\infty}\left(\prod_{l=1}^{j} w_{m+l-1, n+l-1}^{q-2}\right) u_{m+j, n+j}^{q-2} a_{m+j-1, n+j}^{q-1}, \quad m-1, n \geq 0,
$$

which in turn by (3.12) yields the desired result.

\section{Applications involving the classes $\Psi_{d}^{+}$and $\Upsilon_{2 q}^{+}$}

In this section, we present applications of the previous results involving the classes $\Psi_{d}^{+}$and $\Upsilon_{2 q}^{+}$.

Theorem 4.1. Let $q, q^{\prime} \geq 2$ be integers. The following assertions hold:

(i) If a function $\varphi$ belongs to $\Upsilon_{2 q}^{+} \cap \Upsilon_{2 q^{\prime}}$, then $\varphi$ belongs to $\Upsilon_{2 q^{\prime}}^{+}$.

(ii) If a function $\varphi$ belongs to $\left(\Upsilon_{2 q} \backslash \Upsilon_{2 q}^{+}\right) \cap \Upsilon_{2 q^{\prime}}$, then $\varphi$ belongs to $\Upsilon_{2 q^{\prime}} \backslash \Upsilon_{2 q^{\prime}}^{+}$.

Proof. (i) If $q \geq q^{\prime}$, the assertion follows from the inclusion $\Upsilon_{2 q}^{+} \subset \Upsilon_{2 q^{\prime}}^{+}$. So, we may assume that $q<q^{\prime}$. If $\varphi \in \Upsilon_{2 q}^{+}$, Theorem 1.1 in [20] reveals that the $2 q$-Schoenberg sequence of coefficients $\left\{a_{m, n}^{q-2}\right\}_{m, n=0}^{\infty}$ of $\varphi$ has the following property: $\left\{m-n: a_{m, n}^{q-2}>0\right\}$ intersects every arithmetic progression of $\mathbb{Z}$. Taking into account that $\varphi \in \Upsilon_{2(q+1)}$ and the fact that $v_{j, m+1, n}^{q^{\prime}-2}>0$ for all $j$, Theorem 3.2 shows that $a_{m, n}^{q-2}>0$ if and only if $a_{m+j, n+j}^{q-1}>0$, for at least one $j \geq 0$. In particular, the set $\left\{m-n: a_{m, n}^{q-1}>0\right\}$ intersects every arithmetic progression of $\mathbb{Z}$ as well. In other words, $\varphi \in \Upsilon_{2(q+1)}^{+}$, due to Theorem 1.1 in [20] once again. If $q+1=q^{\prime}, \varphi \in \Upsilon_{2 q^{\prime}}^{+}$and we are done. Otherwise, we iterate this procedure until we reach the desired conclusion.

(ii) Assume $\varphi \in\left(\Upsilon_{2 q} \backslash \Upsilon_{2 q}^{+}\right) \cap \Upsilon_{2 q^{\prime}}$. If $\varphi \in \Upsilon_{2 q^{\prime}}^{+}$, then $\varphi \in \Upsilon_{2 q^{\prime}}^{+} \cap \Upsilon_{2 q}$ and $(i)$ would imply that $\varphi \in \Upsilon_{2 q}^{+}$, a contradiction. 
A similar result holds for real spheres with a similar proof. In particular, if $\psi$ belongs to $\Psi_{d} \cap \Psi_{d^{\prime}}^{+}$, then $\psi$ belongs to $\Psi_{d}^{+}$. However, this result was proved earlier in [19, Corollary 1] via a slightly different argument.

Theorem 4.1 allows the following obvious consequences. If $\varphi$ is a function in $\Upsilon_{2 q}$, we write $\varphi_{r}$ to indicate the restriction of $\varphi$ to $[-1,1]$.

Corollary 4.2. For $d \geq 1$ and $q \geq 2$, the following assertions hold:

(i) If a function $\varphi$ belongs to $\Upsilon_{2 q}$ and $\varphi_{r} \circ \cos$ belongs to $\Psi_{d}^{+}$, then $\varphi_{r} \circ \cos$ belongs to $\Psi_{2 q-1}^{+}$.

(ii) If a function $\varphi$ belongs to $\Upsilon_{2 q}^{+}$and $\varphi_{r} \circ \cos$ belongs to $\Psi_{d}$, then $\varphi_{r} \circ \cos$ belongs to $\Psi_{d}^{+}$.

Proof. It suffices to observe that if $\varphi \in \Upsilon_{2 q}$ (respectively, $\Upsilon_{2 q}^{+}$), then $f_{r} \circ \cos \in \Psi_{2 q-1}$ (respectively, $\left.\Psi_{2 q-1}^{+}\right)$and to apply the remark in the paragraph preceding the theorem.

\section{Discussion}

This paper contributes to the literature about the classes $\Psi_{d}, \Upsilon_{2 q}$ and $\Upsilon_{2 q}^{+}$in terms of their Schoenberg sequences. Yet, there are many challenges that involve Schoenberg sequences, for instance in product spaces. Berg and Porcu [7] consider the analogue of Schoenberg pairs introduced in this paper, but on the product space $\mathbb{S}^{d} \times G$, for $G$ a locally compact group. Generalizations of the results in [7] have been provided by [22]. It would be very interesting to inspect whether the results provided in this paper can be generalized to these cases. Another important challenge would be to inspect the Schoenberg pairs related to matrix-valued kernels (see open problem 2 in [39]).

\section{Acknowledgements}

The authors are grateful to the Associate Editor and to the referees for their comments that allowed for an improved version of the manuscript. Research of Valdir A. Menegatto was partially supported by FAPESP, grant 2016/09906-0. Emilio Porcu is partially supported by grant FONDECYT 1130647 from Chilean Ministry of Education, and by Millennium Science Initiative of the Ministry of Economy, Development, and Tourism, grant "Millenium Nucleus Center for the Discovery of Structures in Complex Data".

\section{References}

[1] Abramowitz M., Stegun I.A., Handbook of mathematical functions, with formulas, graphs, and mathematical tables, Dover Publications, Inc., New York, 1966.

[2] Arafat A., Gregori P., Porcu E., Schoenberg coefficients and curvature at the origin of continuous isotropic positive definite kernels on spheres, arXiv:1807.02363.

[3] Baldi P., Marinucci D., Some characterizations of the spherical harmonics coefficients for isotropic random fields, Statist. Probab. Lett. 77 (2007), 490-496, math.PR/0606709.

[4] Barbosa V.S., Menegatto V.A., Strict positive definiteness on products of compact two-point homogeneous spaces, Integral Transforms Spec. Funct. 28 (2017), 56-73, arXiv:1605.07071.

[5] Beatson R.K., zu Castell W., Xu Y., A Pólya criterion for (strict) positive-definiteness on the sphere, IMA J. Numer. Anal. 34 (2014), 550-568, arXiv:1110.2437.

[6] Berg C., Peron A.P., Porcu E., Schoenberg's theorem for real and complex Hilbert spheres revisited, J. Approx. Theory 228 (2018), 58-78, arXiv:1701.07214.

[7] Berg C., Porcu E., From Schoenberg coefficients to Schoenberg functions, Constr. Approx. 45 (2017), 217241, arXiv:1505.05682.

[8] Bingham N.H., Positive definite functions on spheres, Proc. Cambridge Philos. Soc. 73 (1973), $145-156$. 
[9] Chen D., Menegatto V.A., Sun X., A necessary and sufficient condition for strictly positive definite functions on spheres, Proc. Amer. Math. Soc. 131 (2003), 2733-2740.

[10] Christakos G., On certain classes of spatiotemporal random fields with applications to space-time data processing, IEEE Trans. Systems Man Cybernet. 21 (1991), 861-875.

[11] Christakos G., Modern spatiotemporal geostatistics, Oxford University Press, Inc., New York, 2000.

[12] Christakos G., Hristopulos D.T., Bogaert P., On the physical geometry hypotheses at the basis of spatiotemporal analysis of hydrologic geostatistics, Adv. Water Resources 23 (2000), 799-810.

[13] Christakos G., Papanicolaou V., Norm-dependent covariance permissibility of weakly homogeneous spatial random fields, Stoch. Environ. Res. Risk Assess. 14 (2000), 471-478.

[14] Clarke De la Cerda J., Alegría A., Porcu E., Regularity properties and simulations of Gaussian random fields on the sphere cross time, Electron. J. Stat. 12 (2018), 399-426, arXiv:1611.02851.

[15] Daley D.J., Porcu E., Dimension walks and Schoenberg spectral measures, Proc. Amer. Math. Soc. 142 (2014), 1813-1824.

[16] Fiedler J., From Fourier to Gegenbauer: dimension walks on spheres, arXiv:1303.6856.

[17] Gangolli R., Positive definite kernels on homogeneous spaces and certain stochastic processes related to Lévy's Brownian motion of several parameters, Ann. Inst. H. Poincaré Sect. B (N.S.) 3 (1967), 121-226.

[18] Gerber F., Mösinger L., Furrer R., Extending R packages to support 64-bit compiled code: an illustration with spam64 and GIMMS NDVI 3 g data, Comput. Geosci. 104 (2017), 109-119.

[19] Gneiting T., Strictly and non-strictly positive definite functions on spheres, Bernoulli 19 (2013), 1327-1349, arXiv:1111.7077.

[20] Guella J.C., Menegatto V.A., Unitarily invariant strictly positive definite kernels on spheres, Positivity 22 (2018), 91-103.

[21] Guella J.C., Menegatto V.A., Peron A.P., An extension of a theorem of Schoenberg to products of spheres, Banach J. Math. Anal. 10 (2016), 671-685, arXiv:1503.08174.

[22] Guella J.C., Menegatto V.A., Peron A.P., Strictly positive definite kernels on a product of circles, Positivity 21 (2017), 329-342, arXiv:1505.01169.

[23] Guella J.C., Menegatto V.A., Peron A.P., Strictly positive definite kernels on a product of spheres II, SIGMA 12 (2016), 103, 15 pages, arXiv:1605.09775.

[24] Hannan E.J., Multiple time series, John Wiley and Sons, Inc., New York - London - Sydney, 1970.

[25] Hansen L.V., Thorarinsdottir T.L., Ovcharov E., Gneiting T., Richards D., Gaussian random particles with flexible Hausdorff dimension, Adv. in Appl. Probab. 47 (2015), 307-327, arXiv:1502.01750.

[26] Hitczenko M., Stein M.L., Some theory for anisotropic processes on the sphere, Stat. Methodol. 9 (2012), 211-227.

[27] Huang C., Zhang H., Robeson S.M., A simplified representation of the covariance structure of axially symmetric processes on the sphere, Statist. Probab. Lett. 82 (2012), 1346-1351.

[28] Istas J., Spherical and hyperbolic fractional Brownian motion, Electron. Comm. Probab. 10 (2005), 254-262.

[29] Lang A., Schwab C., Isotropic Gaussian random fields on the sphere: regularity, fast simulation and stochastic partial differential equations, Ann. Appl. Probab. 25 (2015), 3047-3094, arXiv:1305.1170.

[30] Leonenko N., Sakhno L., On spectral representations of tensor random fields on the sphere, Stoch. Anal. Appl. 30 (2012), 44-66, arXiv:0912.3389.

[31] Malyarenko A., Invariant random fields on spaces with a group action, Probability and its Applications (New York), Springer, Heidelberg, 2013.

[32] Massa E., Peron A.P., Porcu E., Positive definite functions on complex spheres and their walks through dimensions, SIGMA 13 (2017), 088, 16 pages, arXiv:1704.01237.

[33] Menegatto V.A., Strictly positive definite kernels on the Hilbert sphere, Appl. Anal. 55 (1994), 91-101.

[34] Menegatto V.A., Strictly positive definite kernels on the circle, Rocky Mountain J. Math. 25 (1995), 11491163.

[35] Menegatto V.A., Differentiability of bizonal positive definite kernels on complex spheres, J. Math. Anal. Appl. 412 (2014), 189-199.

[36] Menegatto V.A., Oliveira C.P., Peron A.P., Strictly positive definite kernels on subsets of the complex plane, Comput. Math. Appl. 51 (2006), 1233-1250. 
[37] Menegatto V.A., Peron A.P., Positive definite kernels on complex spheres, J. Math. Anal. Appl. 254 (2001), 219-232.

[38] Møller J., Nielsen M., Porcu E., Rubak E., Determinantal point process models on the sphere, Bernoulli 24 (2018), 1171-1201, arXiv:1607.03675.

[39] Porcu E., Alegria A., Furrer R., Modelling temporally evolving and spatially globally dependent data, Int. Stat. Rev. 86 (2018), 344-377, arXiv:1706.09233.

[40] Porcu E., Bevilacqua M., Genton M.G., Spatio-temporal covariance and cross-covariance functions of the great circle distance on a sphere, J. Amer. Statist. Assoc. 111 (2016), 888-898.

[41] Schoenberg I.J., Positive definite functions on spheres, Duke Math. J. 9 (1942), 96-108.

[42] Trübner M., Ziegel J.F., Derivatives of isotropic positive definite functions on spheres, Proc. Amer. Math. Soc. 145 (2017), 3017-3031, arXiv:1603.06727.

[43] Wünsche A., Generalized Zernike or disc polynomials, J. Comput. Appl. Math. 174 (2005), 135-163.

[44] Ziegel J., Convolution roots and differentiability of isotropic positive definite functions on spheres, Proc. Amer. Math. Soc. 142 (2014), 2063-2077, arXiv:1201.5833. 